\title{
Pathophysiology of parkinsonism due to hydrocephalus
}

\author{
B A Racette, G J Esper, J Antenor, K J Black, A Burkey, S M Moerlein, T O Videen, V Kotagal, \\ J G Ojemann, J S Perlmutter
}

J Neurol Neurosurg Psychiatry 2004;75:1617-1619. doi: 10.1136/jnnp.2003.028449

We report a patient with hydrocephalus who developed levodopa responsive parkinsonism and severe bradyphrenia associated with shunt malfunction and revision. Magnetic resonance imaging revealed periaqueductal edema involving medial substantia nigra. $\left[{ }^{18} \mathrm{~F}\right] \mathrm{dopa}$ positron emission tomography demonstrated reduced uptake in the caudate and putamen with relative sparing of the posterior putamen. Hydrocephalus associated with shunt malfunction can cause a distinct parkinsonian syndrome with greater dysfunction of projections from the medial substantia nigra to anterior striatum than in idiopathic Parkinson's disease.

C ommunicating and non-communicating hydrocephalus can produce parkinsonism that may be relieved with ventricular shunt placement. ${ }^{1}$ Clinical features include bradykinesia, rest and postural tremor, loss of postural reflexes, rigidity, and bradyphrenia. Signs of dorsal midbrain impairment such as lid retraction, convergence-retraction nystagmus, light-near dissociation, and forced downgaze may be present. ${ }^{1}$ These atypical features suggest that parkinsonism due to hydrocephalus is pathophysiologically distinct from Parkinson's disease (PD). Physical factors such as increased ventricular pressure near the upper midbrain and diencephalon may cause shear, torsion, and ischaemia of projections to the striatum or direct involvement of the striatum. ${ }^{1}$ We used magnetic resonance imaging (MRI) and $6-\left[{ }^{18} \mathrm{~F}\right]$ fluorodopa ( $\left.\left[{ }^{18} \mathrm{~F}\right] \mathrm{dopa}\right)$ positron emission tomography (PET) to investigate the pathophysiology of parkinsonism associated with hydrocephalus and shunt malfunction.

\section{PATIENT AND METHODS}

\section{Patient}

This patient was a 44 year old right handed man who presented at age 26 with personality change and headaches and was diagnosed as having hydrocephalus due to aqueductal stenosis. The placement of a ventriculoperitoneal shunt alleviated his symptoms. At age 43 he developed headaches, episodic confusion, and lethargy that were improved by shunt revision.

One month prior to admission, he developed headaches, personality change, and disorientation. Ventricular catheter revision provided modest improvement in symptoms. Within 10 days, he developed persistent downward gaze, headaches, bradykinesia, and reduced speech production. Head computed tomography (CT) showed stable ventricular size. Radiographic visualisation of the shunt hardware showed no disconnection, and he was transferred to our institution for further management.

Examination revealed markedly slow response time and paucity of spontaneous speech. He was completely oriented. He perseverated and provided one word answers. His voice was severely hypophonic and nasal. He had lid retraction and convergence-retraction nystagmus but no light-near dissociation. He had slightly asymmetric rigidity and bradykinesia, severe akinesia, and $3 \mathrm{~Hz}$ rest/action tremor in both hands. He had severe postural instability and was unable to walk unassisted. Plantar reflexes were extensor. Unified Parkinson's Disease Rating Scale motor subscale 3 (UPDRS $3),{ }^{2}$ was 100 out of a possible 108 (on no PD medications).

Ventricular shunt opening pressure was zero. Cerebrospinal fluid studies were normal. A radionuclide study confirmed shunt function.

Levodopa/carbidopa (1500 mg/375 mg per day) markedly improved verbal response time, bradykinesia, and rigidity. With treatment he walked independently but had residual postural instability. No quantitative measures of verbal response time were collected but response time improved from several minutes prior to treatment to seconds after treatment. With treatment and when "on", UPDRS 3 score was 6.5/108. To investigate the pathophysiology of his parkinsonism, a 3 tesla MRI and $\left[{ }^{18} \mathrm{~F}\right]$ dopa PET scan were performed.

\section{Methods}

These studies were approved by the Human Studies Committee and the Radioactive Drug Research Committee of Washington University School of Medicine. All participants gave written informed consent.

\section{$\left[{ }^{18} \mathrm{~F}\right]$ dopa PET studies}

$\left[{ }^{18} \mathrm{~F}\right]$ dopa PET scans were performed in the subject (age 44) and in 12 age matched controls (six women, six men; mean age 45 (SD 3.7) years) with typical idiopathic PD. The PD controls had disease duration of 2 (SD 1.1) years and UPDRS3 mean 18 (SD 7) (off medications). We also did $\left[{ }^{18} \mathrm{~F}\right]$ dopa PET studies on 11 normal control subjects (four men, seven women; mean age 49 (11) years) who had normal neurological exams. The subjects were prepared and PET scans were collected, reconstructed, and aligned as previously described. ${ }^{3}$ A Siemens 953b in 3D mode was used for all PET studies. Reconstructed resolution of the images was about $6 \mathrm{~mm}$ FWHM in the transverse plane.

\section{5-T MRI}

Each subject had a 3-D MPRAGE MRI with a Siemens Magnetom Vision 1.5 T scanner. A reviewer blinded to the subjects' clinical status outlined the entire caudate, anterior and posterior putamen, and an occipital region on each side of the brain.

The MPRAGE image was co-registered to the $\left[{ }^{18} \mathrm{~F}\right]$ dopa PET image using $\mathrm{AIR}^{4}$ and this transformation matrix was used to resample the MR identified regions of interest in the PET image. Decay corrected regional tissue activity PET measurements were extracted from the dynamic series of PET

Abbreviations: $\left[{ }^{18} \mathrm{~F}\right]$ dopa PET, 6- $\left[{ }^{18} \mathrm{~F}\right]$ fluorodopa positron emission tomography; MRI, magnetic resonance imaging; PD, Parkinson's disease; UPDRS, Unified Parkinson's Disease Rating Scale 
images, and an influx constant (Ki) was calculated with the occipital region as the input using data from 24 minutes to 94 minutes after injection. ${ }^{56}$

\section{3-T MRI}

To delineate the periaqueductal structures, the subjects underwent a Siemens Allegra 3.0-T MRI scan to obtain turbo spin echo (repetition time $(\mathrm{TR})=7000 \mathrm{~ms}$; echo time $(\mathrm{TE})=96 \mathrm{~ms}$ ) and fluid attenuated inversion recovery (FLAIR; coronal acquisition: $\mathrm{TR}=7000, \mathrm{TE}=110$, inversion time $(\mathrm{TI})=2100)$ images.

\section{RESULTS}

$\left[{ }^{18} \mathrm{~F}\right]$ dopa PET uptake in our patient was reduced in both the caudate and putamen relatively similarly, which was different from typical idiopathic PD controls (table 1). Although the absolute reduction in the caudate and putamen in our patient was similar to that in the typical idiopathic PD controls, we found that the ratio of caudate to posterior putamen Ki for $\left[{ }^{18} \mathrm{~F}\right]$ dopa in our patient was below the range found in the idiopathic PD controls (see table 1).

The 3-T MRI demonstrated increased signal in the region of the ventricular system and the surrounding brain structures on the TSE image (fig lA, B). No PD control had increased signal in this region.

\section{DISCUSSION}

In idiopathic $\mathrm{PD}$, there is a characteristic pattern of dopamine cell loss in the substantia nigra with more lateral areas affected earlier and less degeneration of more medial regions. ${ }^{7}$ These lateral neurones preferentially project to the posterior putamen explaining the preferential loss of $\left[{ }^{18} \mathrm{~F}\right]$ dopa uptake in the posterior putamen in PD. This may explain the apparent motor manifestations early in the disease since the posterior putamen more than anterior striatal regions affects motor control. ${ }^{8}$ Our patient shares many features with idiopathic PD including the motor manifestations and dramatic response to levodopa. The reduced uptake of $\left[{ }^{18} \mathrm{~F}\right]$ dopa in the putamen is consistent with these clinical observations. Although we cannot prove that our patient's parkinsonism was due to the hydrocephalus or shunt revision, the abrupt onset, atypical features, and relatively greater caudate: putamen loss are atypical of PD or other degenerative parkinsonisms.

Our patient had marked, levodopa responsive bradyphrenia that was dramatically different from PD. Bradyphrenia in PD may be due to dopamine deficiency; however, the motor manifestations may be dissociated and it is difficult to separate completely slowed cognitive processing from motor slowing. ${ }^{9}$ The absolute loss in the caudate is not a likely contributing factor since this was not different between our patient and the PD controls. There are two other factors that may contribute to these behavioural abnormalities: the relatively greater dysfunction of the caudate compared with the posterior putamen and the relatively rapid loss of
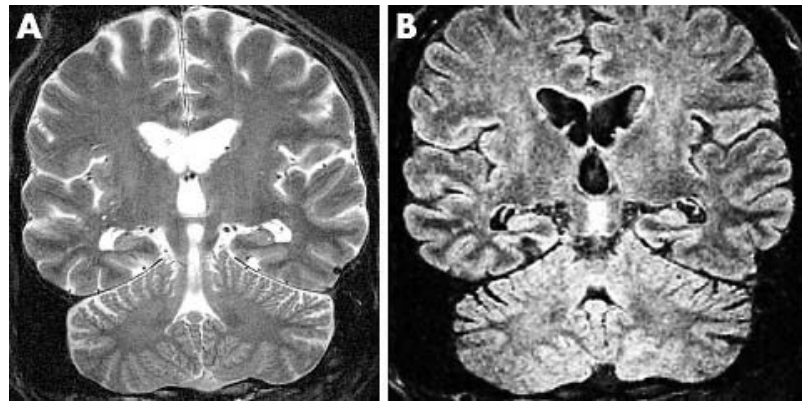

Figure 1 (A) 3-T MRI of the brain in the patient with hydrocephalus demonstrating increased signal in the region of the ventricular system and the surrounding brain structures on the TSE image. Flow was evident through the aqueduct (images not shown). (B) FLAIR sequence, which suppresses the signal from fluid filled spaces, reveals that the increased signal comes from the diencephalic and midbrain tissues adjacent to the ventricular system.

function in the caudate as compared with the average person with idiopathic PD. Our data demonstrate that the caudate to putamen ratio was below the range that we found in PD. However, the difference in the rate of loss in our patient and $\mathrm{PD}$ is speculation based upon the abrupt development of clinical manifestations since we have no longitudinal neuroimaging data. Nevertheless, the rate of loss may be an important factor in the severity of this patient's clinical manifestations despite the relatively modest defects on $\left[{ }^{18} \mathrm{~F}\right]$ dopa PET. ${ }^{10}$

Neuroimaging studies provide important clues to the pathophysiology of our patient's condition. The reduced uptake of $\left[{ }^{18} \mathrm{~F}\right]$ dopa throughout the striatum indicates either direct impairment of the striatum (perhaps from the effects of enlarged lateral ventricles), interruption of the nigrostriatal pathways or dysfunction of the substantia nigra pars compacta. These findings are consistent with single photon emission computed tomography (SPECT) studies that demonstrate reduced cerebral blood flow in both the caudate and putamen ${ }^{11}{ }^{12}$; however, the resolution of SPECT is not as good as PET and $\left[{ }^{18} \mathrm{~F}\right]$ dopa is a more direct measure of basal ganglia function. Hydrocephalus could directly impair caudate since the caudate nuclei sit adjacent to the lateral ventricles, but this would not explain putaminal dysfunction. The FLAIR MRI provides an important clue to the site of pathology. Markedly increased signal in the dorsal and ventral medial midbrain suggests that there could be preferential dysfunction of the medial aspects of the substantia nigra pars compacta. This abnormality could be due to direct pressure from the hydrocephalus or shear forces from shunt revision with subsequent changes in ventricular pressure in that area. Although not directly visualised, it is possible that these same forces produce mechanical disruption of nigrostriatal projections including those fibres from

Table $1 \quad\left[{ }^{18} \mathrm{~F}\right] \mathrm{dopa}$ influx constants $(\mathrm{Ki})^{\star}$ in the patient and the controls with Parkinson's disease (PD)

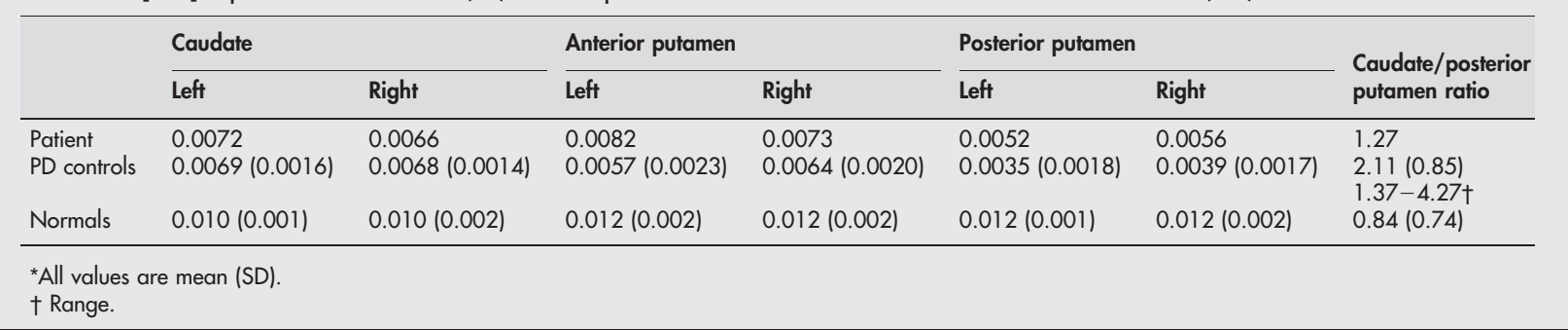


the medial substantia nigra. The increased signal on FLAIR may indicate residual (or new) edema that may explain the lack of symptom resolution with shunt revision. Direct dorsal midbrain impairment of medial structures including the posterior commissure and rostral interstitial nucleus of the medial longitudinal fasciculus due to the shunt revision likely produced his eye movement abnormalities. Failure of the parkinsonism to resolve with shunt revision might have been due to intermittent shunt dysfunction not detected with one time measurement of shunt pressure. Alternatively, there might be a critical duration of shunt malfunction that leads to irreversible brain injury. Although parkinsonism due to non-communicating hydrocephalus has been well described, this report provides possible anatomical and pathophysiological clues to this illness.

\section{ACKNOWLEDGEMENTS}

We thank Dr Steve Lee for help with the patient.

\section{Authors' affiliations}

B A Racette, G J Esper, J Antenor, K J Black, A Burkey, T O Videen, V Kotagal, J S Perlmutter, Department of Neurology and Neurological Surgery (Neurology), Washington University School of Medicine, St Louis, MO, USA

B A Racette, K J Black, J S Perlmutter, American Parkinson Disease Association Advanced Center for Parkinson Research, Washington University School of Medicine, St Louis, MO, USA

B A Racette, K J Black, J S Perlmutter, Huntington Disease Society of America Center of Excellence, Washington University School of Medicine, St Louis, MO, USA

K J Black, S M Moerlein, J S Perlmutter, Mallinckrodt Institute of Radiology, Washington University School of Medicine, St Louis, MO, USA

J G Ojemann, J S Perlmutter, Department of Anatomy and Neurobiology, Washington University School of Medicine, St Louis, MO, USA

J G Ojemann, J S Perlmutter, Department of Neurology and Neurological Surgery (Neurosurgery), Washington University School of Medicine, St Lovis, MO, USA

This work was supported by NIH grants K23NS43351, NS41509, the Greater St. Lovis Chapter of the American Parkinson Disease
Association, the Barnes-Jewish Hospital Foundation (The Jack Buck Fund), the Sam \& Barbara Murphy Fund, the Elliot H Stein Family Fund and a donation from Ruth Kopolow.

Competing interests: none declared

Correspondence to: $\operatorname{Dr} B$ A Racette, Washington University School of Medicine, 660 South Euclid Ave, Box 8111, St. Louis, MO 63110; racetteb@neuro.wustl.edu

Received 16 September 2003

In revised form 6 February 2004

Accepted 20 February 2004

\section{REFERENCES}

1 Curran T, Lang AE. Parkinsonian syndromes associated with hydrocephalus: case reports, a review of the literature, and pathophysiological hypotheses. Mov Disord 1994:9:508-20.

2 Fahn S, Elton RL. Members of the UPDRS Development Committee. Unified Parkinson's disease rating scale. In: Fahn S, Marsden CD, Goldstein M, Calne DB, eds. Recent developments in Parkinson's disease. New York: Macmillan, 1987:153-63.

3 Racette BA, McGee-Minnich L, Moerlein SM, et al. Welding-related parkinsonism: clinical features, treatment, and pathophysiology. Neurology 2001;56:8-13.

4 Woods RP, Mazziotta JC, Cherry SR. MRI-PET registration with automated algorithm. J Comput Assist Tomogr 1993;14:536-46.

5 Patlak CS, Blasberg RG, Fenstermacher JD. Graphical evaluation of blood-tobrain transfer constants from multiple-time uptake data. J Cereb Blood Flow Metab 1983;3:1-7

6 Logan J, Wolf AP, Shive C-Y, et al. Kinetic modeling of receptor-ligand binding applied to positron emission tomographic studies with neuroleptic tracers. J Neurochem 1987;48:73-83.

7 Damier P, Hirsch EC, Agid Y, et al. The substantia nigra of the human brain. II. Patterns of loss of dopamine-containing neurons in Parkinson's disease. Brain 1999; 122(Pt 8): 1437-48.

8 Nambu A, Kaneda K, Tokuno H, et al. Organization of corticostriatal motor inputs in monkey putamen. J Neurophysiol 2002;88:1830-42.

9 Rafal RD, Posner MI, Walker JA, et al. Cognition and the basal ganglia. Separating mental and motor components of performance in Parkinson's disease. Brain 1984; 107:1083-94.

10 Ishikawa T, Dhawan V, Chaly T, et al. Clinical significance of striatal DOPA decarboxylase activity in Parkinson's disease. J Nucl Med 1996;37:216-22.

11 Shahar E, Lambert R, Hwang PA, et al. Obstructive hydrocephalus-induced parkinsonism. I: Decreased basal ganglia regional blood flow. Pediatr Neurol 1988:4:117-19.

12 Aggarwal S, Childers MK, Jimenez D. Use of carbidopa-levodopa in a patient with hydrocephalus and frozen movement. Brain Inj 1997;1 1:831-6. 